\title{
Medical Robot with Electronic Health Record System
}

Pruthviraj RD ${ }^{1^{*}}$, Subhash $\mathrm{KC}^{2}$, Namratha $\mathrm{K}^{2}$ and Sushma $\mathrm{KR}^{2}$

${ }^{1}$ Department of Engineering Chemistry, Amruta institute of Engineering and Management Sciences, Bidadi, Bangalore, India

${ }^{2}$ Department of Electronics and Communication Engineering, Amruta institute of Engineering and Management Sciences, Bidadi, Bangalore, India

"Corresponding author: Pruthviraj RD, Department of Engineering Chemistry, Amruta institute of Engineering and Management Sciences, Bidadi, Bangalore, India, Tel : 7846868398; E-mail: namrathakantharaj@gmail.com

Rec Date: Apr 21 2014; Acc Date: May 21 2014; Pub Date: May 232014

Copyright: (c) 2014 Pruthviraj RD, et al. This is an open-access article distributed under the terms of the Creative Commons Attribution License, which permits unrestricted use, distribution, and reproduction in any medium, provided the original author and source are credited.

\begin{abstract}
The healthcare systems require constant monitoring of patients and their records for speedy recovery and also to change the course of tablets at different stages. Most of the healthcare systems are found inefficient in their performance due to human errors in the monitoring of patients who are in the critical care units where human intervention is limited. The obvious reason would also be that the records i.e. the history of the patient may not be available before hand and the doctor will be misguided to prescribe wrong medicines and wrong treatment. Hence, we have come up with the concept of Medical Robot with Electronic health record system-a bridge between the man and the machine, to reduce these errors for effective performance.
\end{abstract}

\section{Introduction}

The concept of creating machines that can operate autonomously dates back to classical times, but research into the functionality and potential uses of robots did not grow substantially until the 20th century [1]. Throughout history, robotics has been often seen to mimic human behaviour, and often manage tasks in a similar fashion. Today, robotics is a rapidly growing field, as technological advances continue; research, design, and building new robots serve various practical purposes, whether domestically, commercially, or militarily. Many robots do jobs that are hazardous to people such as defusing bombs and mines [2].

Today, robotic devices are used to replace missing limbs, perform delicate surgical procedures, and deliver neuro rehabilitation therapy to stroke patients, teach children with learning disabilities, and perform a growing number of other health related tasks [3].

Here is one such concepts which provides the best possible solution for the challenges faced by humans in the field of medicine as shown in the section 1

We have divided the paper into 3 main sections.

Section 1: Problem Statement.

Section 2: Solution - The concept of Medical robot with EHRS.

Section 3: Conclusion.

\section{Problem Statement}

We can see huge number of hospitals using the robots for surgical purposes which are giving very good results. But, most of the people do suffer a lot even before the surgery without proper medication, improper method of maintaining patients' record and few human errors [4].

These were the observations from the hospitals which are in rural parts of the country.

\section{Patients suffer due to lack of proper medication leading to death in many cases}

Medicine is not "one size fits all." Some medications may trigger allergies, while others may react negatively with previously prescribed drugs. If such risks are ignored, patients can experience severe allergic reactions, major health problems or death.

\section{Identification/information of the patient may not be sufficient/accurate}

Patient identification and the matching of a patient to an intended treatment is an activity that should be performed routinely in all care settings. Risks to patient safety occur when there is a mismatch between a given patient and components of their care, whether these components are diagnostic, therapeutic or supportive [5]. Throughout health care, the failure to correctly identify patients and match that information to an intended clinical intervention continues to result in wrong person, wrong site procedures, medication errors, transfusion errors and diagnostic testing errors.

\section{Records may be abused by the third party for illegal reasons}

Chances of abusing the medical records of patients for illegal purposes are very high if it is not secured properly.

\section{Illiteracy leads individuals to report non-accurate identity}

Illiterate people face many problems in understanding what is written on the records. Most of them faces problem in using ID cards provided by hospitals.

\section{Lack of automation in hospitals}

For Hospitals, it is essential to keep constant touch with the staff, patients, utility service provider and suppliers to maintain best standards of the healthcare services [6]. 
All the above mentioned factors lead to serious consequences on the entire Human Community.

\section{Solution - The Concept of Medical Robot with EHRS}

We have come up with a solution to all the above mentioned problems. Burden on the humanity community can be reduced to a very large extent [7]. A robot which can serve patients on time which can be programmed so as to store the entire data of a patient like: disease, name of the tablets, timings at which the tablets should be provided, X-ray report, CT scan report and other types of reports which describes the flaw in the body of human [8].

The robot can also traverse to the other rooms of the hospital in order to serve different patients suffering from various diseases.

\section{EHRS (Electronic Health Record System)}

EHRS is software which is also termed as electronic patient record (EPR) or computerized patient record. It is an evolving software concept defined as a systematic collection of electronic health information about individual patients or populations. It is a record in digital format that is capable of being shared across different health care settings, by being embedded in network-connected enterprisewide information systems [9]. Its purpose can be understood as a complete record of patient encounters that allows the automation and streamlining of the workflow in health care settings and increases safety through evidence-based decision support, quality management, and outcomes reporting.

\section{Design}

The front end of EHRS is completely designed using Microsoft Visual basic 6 and is backed up with the MYSQL Database. The EHRS comes in 2 versions.

- Standalone Electronic Health Record System (S-EHRS)

- This version of EHRS does not require any type of network connections such as LAN, WAN, etc.as it has built in database to store and retrieve the data. These types are useful when there is a network error.

- Consolidate Electronic Health Record System (C-EHRS)

- C-EHRS finds its application on the Medical robot while communicating with the server.

\section{Brief working}

- The software will be initiated when there is input from RFID Reader to the serial port.

- Now it compares weather the detected RFID tag is valid or not with the data that are included in Database.

- If the Detected RFID tag is a valid one, the software shows the complete details about the RFID tag owner (i.e. Patient). Else, some other action will be taken.

- If the prescription is to dispatch the tablets, then the software goes on checking the Column-"No. of Tablets" and sends a certain no. of bits (it depends on the value given in that column and No. of bits assigned to that Value) from the Serial port which is connected to a Microcontroller Board [Arduino board (ATMEGA8)]. Then the further actions are carried out by Microcontroller.
- The C-EHRS enables robot to retrieve the database from other computer (server) via Wi-Fi network. All the Databases are Password Protected and can be accessed only by robot and the respective doctors.

- The software enables telemedicine facility using Skype protocols when necessary.

\section{Medical robot}

Robots are perfect machinery devices which are used to reduce the burden and risks. The robot here is completely embedded with the EHRS and works according to the signals received from the EHRS [10]. This robot is capable of locomotion from one patient to another patient in order to serve the patients as prescribed by the doctors. It is also capable of storing, retrieving the patients data in presence of doctors.

\section{Control system}

The control system of Medical robot includes microcontrollers and EHRS software. A microcontroller is a small computer on a single integrated circuit containing a processor core, memory, and programmable input/output peripherals [11]. There are varieties of microcontrollers; in this project, we are using ATMEGA8 to control the locomotion of the robot and ATMEGA328 to work with the EHRS. They were programmed using Arduino IDE.

\section{External devices}

The devices that we are using in this project are DC Motors.

They are used to loco mote the robot, and to perform some other tasks that are given by the micro controllers [12].

\section{Amplifiers}

In this project, we are using ULN2003AN (7-Darlington emitter follower) IC as a Current amplifier. It amplifies the input current that are produced by the Line Sensors and feed the amplified output to ARDUINO board (Figure 1).

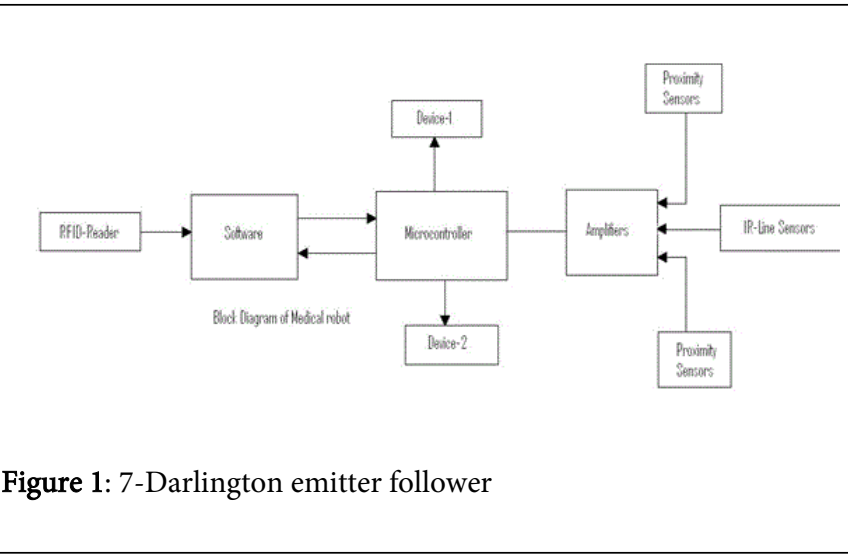

\section{Sensors}

A sensor is a device that measures a physical quantity and converts it into a signal which can be read by an observer or by an instrument [13]. Sensors like Proximity sensors, Line tracking sensors, RFID Readers are used in this project 
- A proximity sensor is a sensor able to detect the presence of nearby objects without any physical contact. Here, we are using IR Proximity sensors; these sensors help the robot to detect any object / person in front of the robot and takes decision according to the situation.

- An RFID reader is a device that is used to interrogate an RFID tag. These are the radio frequency identifying sensors which are used for authentication of patients, doctors, or any other users. The RFID tag talks to the interrogator using what is called the air- interface. This is a specification for how they talk to each other and includes the frequency of the carrier, the bit data rate, the method of encoding. ISO 18000 is the standard for the air interface for item management.

- Line Tracking Sensors are mainly used to track the marked lines. It may be either Black line on a bright background or White line on a Dark background. Usually Line tracking sensors are made up of Photodiodes and IR LED (Figure 2).

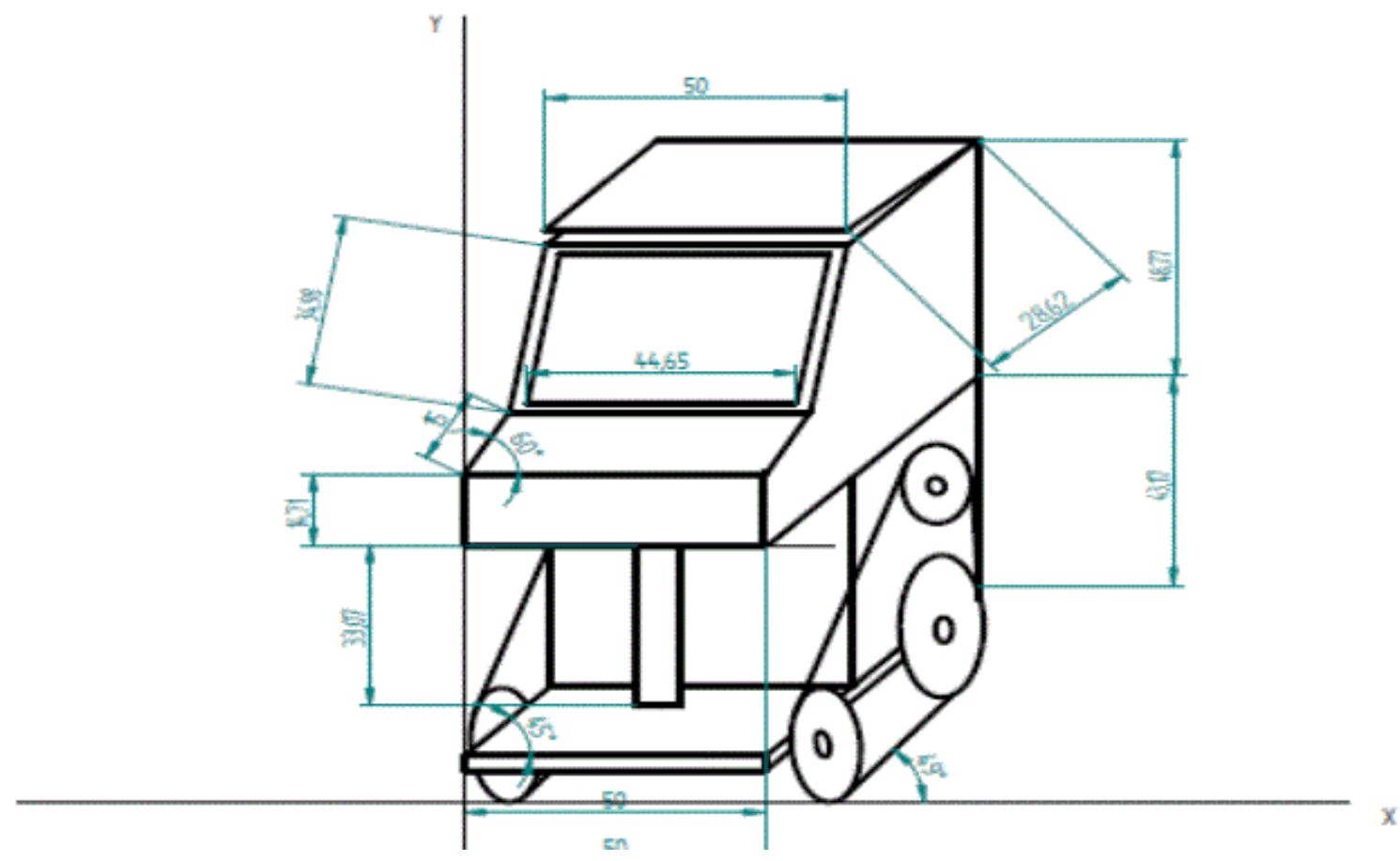

Figure 2: CAED Diagram of the Medical Robot

\section{The Robot's structure}

The robot's body is designed in such a way as to maintain its stability even when there is more weight. The locomotion is entirely controlled by the DC Motors having the torque of 78.4532 newton centimeter at the speed 300RPM [14]. The locomotion of the robot is gripped with the rubber track belts to maintain very good friction even on the soft surface.

\section{Conclusion}

The EHRS software enables the designed robot to supply the prescribed tablets and treatment synopsis to the respective patients regardless of the liking of the patient and it effectively carries out the orders given by the doctor. It also allows wireless communication between the doctor and the patient saving both time and expenditure.

\section{Special features}

- Wireless communication between patients and doctors whenever necessary.

- The data base is secured and can be accessed by doctors only when they are authenticated.
- Telemedicine facility in case of emergency.

- On spot diagnosis option.

- The EHRS can switch between 2 different modes. Standalone mode when there is a proper connection between the robot and the server Consolidate mode when there is a problem in communicating with the server.

- Can be used with any type of electro-medical equipment.

Hence, this concept is proved efficient in utilization of machines for effective performance of the healthcare centres thereby providing solution to one of the global problem.

This project was shortlisted as Top 10 Finalists in the IEEE Region 10's All India Young Engineers' Humanitarian Challenge-2011. 
Citation: Pruthviraj RD, Subhash KC, Namratha K, Sushma KR (2014) Medical Robot with Electronic Health Record System. Adv Robot Autom 3: 119. doi:10.4172/2168-9695.1000119

Page 4 of 4

\section{The final design}

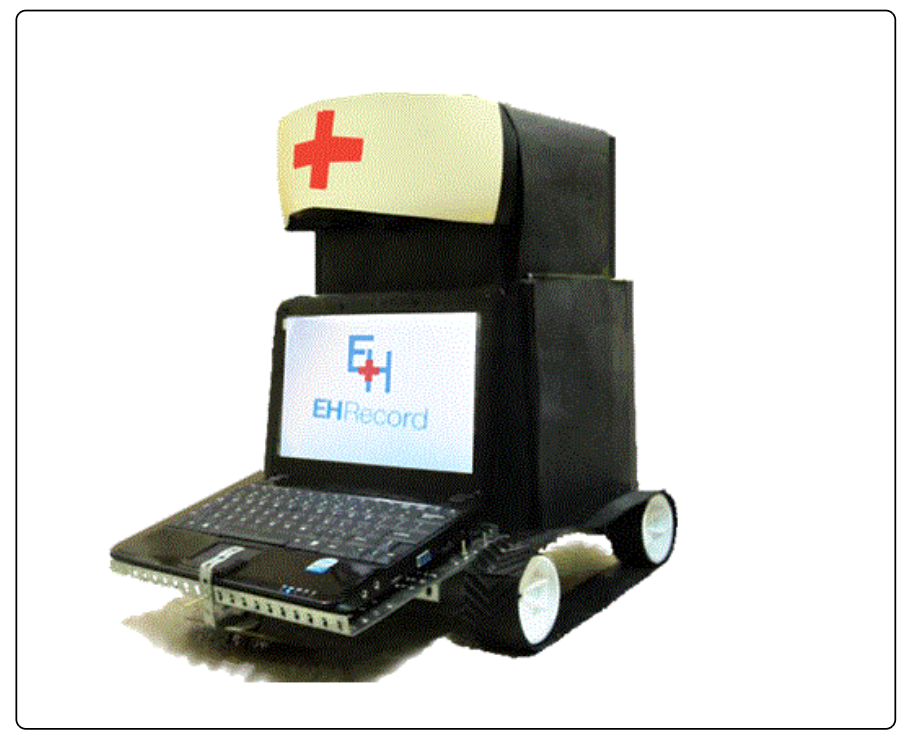

\section{References}

1. http://spectrum.ieee.org/robotics/medical-robots

2. www.doc.ic.ac.uk/ nd/surprise_96/journal/vol4/ao2/report.html

3. http://www.ismp.org/Tools/guidelines/ADC_Guidelines_Final.pdf

4. http://www.pppmag.com/documents/V3N6/WDIToT_Pg32.pdf

5. http://en.wikipedia.org/wiki/Arduino

6. http://en.wikipedia.org/wiki/Visual_studio

7. http://en.wikipedia.org/wiki/Multiplexer

8. http://pdf.datasheetcatalog.com/datasheet/fairchild/DM74157.pdf

9. http://www.doyoung.net/video/DATASHEET/PDF/ULN2003.pdf

10. http://users.ece.utexas.edu/ valvano/Datasheets/L293d.pdf

11. http://www.bigresource.com/VB-RFID-programming-wePC2boqvb.html

12. http://www.nejm.org/doi/full/10.1056/NEJMsb1205420\#t=article

13. http://jamia.bmj.com/content/16/4/457

14. http://jamia.bmj.com/content/20/1/144.full.pdf+html 